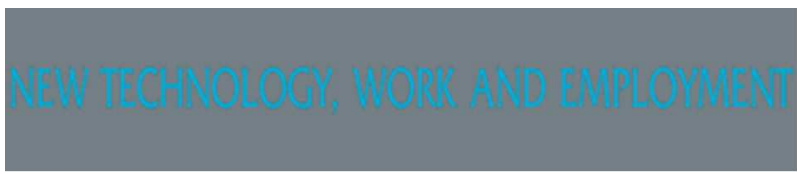

\title{
Workplace Stress from Actual and Desired Computer- Mediated Communication Use: A Multi-Method Study
}

\begin{tabular}{|c|c|}
\hline Journal: & New Technology, Work and Employment \\
\hline Manuscript ID & MA-NTWE-Jun-2015-045.R3 \\
\hline Wiley - Manuscript type: & Main Article \\
\hline Keywords: & $\begin{array}{l}\text { Computer-Mediated Communication, Email, Instant messaging, } \\
\text { Technostress, Workplace stress, Workload, Wellbeing, Misfit, Person- } \\
\text { Environment fit, Multi-method study }\end{array}$ \\
\hline Abstract: & $\begin{array}{l}\text { The use of computer-mediated communication applications can lead to } \\
\text { workplace stress for employees. However, such stress is influenced not } \\
\text { only by how individuals actually use computer-mediated communication } \\
\text { applications, but also how they desire to use them. This paper examines } \\
\text { how the individual's actual and desired use of communication tools } \\
\text { together influence his or her workplace stress. It does so across a range of } \\
\text { computer-mediated media (e.g. email or instant messaging), and } \\
\text { workplace stressors (e.g. workload or work relationships). This } \\
\text { investigation is conducted using a multi-method research design. The } \\
\text { quantitative study found that desired and actual use together influenced } \\
\text { workplace stress, mostly for email, but not for other media. The qualitative } \\
\text { study further showed that such influence depends on organizational } \\
\text { conditions such as available media or co-workers preferences. The findings } \\
\text { emphasize the importance of considering the individuals' desired use of } \\
\text { CMC media, and their subjective appraisals of different media. }\end{array}$ \\
\hline
\end{tabular}

\section{SCHOLARONE $^{\text {ix }}$




\title{
Workplace Stress from Actual and Desired Computer-Mediated Communication Use: A Multi-Method Study
}

\begin{abstract}
:
The use of computer-mediated communication applications can lead to workplace stress for employees. However, such stress is influenced not only by how individuals actually use computer-mediated communication applications, but also how they desire to use them. This paper examines how the individual's actual and desired use of communication tools together influence his or her workplace stress. It does so across a range of computer-mediated media (e.g. email or instant messaging), and workplace stressors (e.g. workload or work relationships). This investigation is conducted using a multi-method research design. The quantitative study found that desired and actual use together influenced workplace stress, mostly for email, but not for other media. The qualitative study further showed that such influence depends on organizational conditions such as available media or co-workers preferences. The findings emphasize the importance of considering the individuals' desired use of CMC media, and their subjective appraisals of different media.
\end{abstract}

Keywords: computer-mediated communication, email, instant messaging, technostress, workplace stress, workload, work relationships, wellbeing, misfit, person-environment fit, multi-method study 


\section{INTRODUCTION}

The introduction of computers in the workplace has transformed interpersonal communications. Potential negative consequences of their use have attracted considerable attention from practitioners and academics. Specifically, the use of computer-mediated communication $(\mathrm{CMC})$ has been investigated as a potential cause of workplace stress for individuals. Studies have found that $\mathrm{CMC}$ use increases work demands, leading to work overload (Barley et al., 2011), work-life conflict (Stich et al., 2015), burnout, and psychological distress (Barber and Santuzzi, 2015; Mano and Mesch, 2010). Email applications for instance are often found to be overwhelming in the volume of email they generate (Dabbish and Kraut, 2006; Mano and Mesch, 2010).

However, while use of $\mathrm{CMC}$ can potentially increase workplace stress, the appraisal of stress is individual-specific. For instance, not every employee who experiences work-life conflict attributes it to the use of corporate smartphones. Indeed, some actually perceive smartphones as part of their overall lifestyle. In such a case, they experience less work-life conflict (Derks et al., 2016), and feel a greater sense of professionalism (Cavazotte et al., 2014). Moreover, the same extent of CMC use may be perceived differently by different individuals (Higgins et al., 1985), and individuals having a positive attitude toward email feel less stressed by them (Sumecki et al., 2011). Every individual does not experience workplace stress from the use of CMC to the same extent, and in the same way. It is thus important to take into account the individual's subjectivities regarding the use of CMC media, when investigating the latter's influence on workplace stress.

These examples suggest that the influence of CMC use on workplace stress is influenced both by how individuals actually use $\mathrm{CMC}$ and how they desire to do so. However, the simultaneous influence of these two factors has not been investigated systematically in 
empirical studies or literature reviews. Do actual CMC use and desired CMC use influence workplace stress together? If so, which workplace stressors are affected and how? Is this influence the same for every communication medium? The lack of answers to these questions is theoretically and practically problematic because it leaves a gap in our understanding of how and why an individual may or may not experience workplace stress due to CMC use. The present research has the aim, therefore, to explore how individuals' 'desired use' of CMC influences the relationships between CMC use and workplace stress. Specifically, we address the following two research questions:

Research Question 1: To what extent do actual and desired computer-mediated communication use together, affect workplace stress experienced by individuals?

Research Question 2: How do actual and desired computer-mediated communication use together, affect workplace stress experienced by individuals?

A multi-method design that includes quantitative and qualitative data is used to investigate these research questions (Creswell and Clark, 2011). The first study uses quantitative data, and examines the extent to which actual and desired CMC use together influence workplace stress. The second study uses qualitative data, to explore how and why individuals experience workplace stress are due to both their actual and desired CMC use. Both studies' purposes, samples, methods and results are presented separately, with a final part merging their respective discussions. The paper contributes to the theoretical understanding of workplace stress due to $\mathrm{CMC}$ use. It emphasizes the importance of taking into account the individual's preferences in articulating the relationship between CMC use and workplace stress. 
The paper is organized as follows. In the next section, we present literature on the influence of actual and desired CMC use on workplace stress. Section 3 presents the study's mixedmethod research approach. The quantitative and qualitative studies' objectives, data and results are discussed in Sections 4 and 5 respectively. An integrative discussion is presented in Section 6, along with a summary of contributions and implications of the research.

\section{THE INFLUENCE OF ACTUAL AND DESIRED COMPUTER-MEDIATED COMMUNICATION USE ON WORKPLACE STRESS}

In this section we first present the literature discussing the influence of the extent of CMC use on workplace stress. We then describe studies that have considered how workplace stress could potentially be influenced by actual and desired CMC together.

\subsection{Computer-mediated communication use and workplace stress}

Stress is defined as the process by which individuals appraise demand conditions in the environment as stressors, activate coping behaviors, and experience varying levels of resulting strain (Cooper et al., 2001). CMC use has been considered as a potential source of workplace stress. Employees who communicate with each other using CMC could face various demands, such as pressures to respond quickly to incoming messages, expectations of constant availability, increased workload due to CMC or relationship problems like misunderstandings or cyberbullying (Stich et al., 2015). These demands influence diverse workplace stressors such as work-life conflict and work overload, resulting in strain outcomes such as distress, burnout and anxiety (Stich et al., 2015).

The literature qualifies CMC use in a number of ways. The first is to consider the volumes of interactions and messages. Studies thus focus on the 'amount' or 'extent' of communication or time spent using the CMC application. For example, the higher the volume of email sent and 
received, the higher the feelings of email stress (Mano and Mesch, 2010) and email overload (Sumecki et al., 2011). The second is to consider the time spent in handling or managing CMC communication; greater the time, the higher the feelings of work overload (Barley et al., 2011). Thirdly, studies focus on the characteristics of different CMC applications. Characteristics such as asynchronicity and lack of visual and emotional cues make it more likely to escalate disputes due to ambiguity of content, leading to potential miscommunication (Byron, 2008). CMC use may thus cause workplace stress because of some intrinsic characteristics of media. Many of these studies have however been criticized for their focus on a technologically deterministic approach (Dén-Nagy, 2014, p. 196) because they do not consider the individual's subjective appraisal of the stress creating CMC use situation.

\subsection{Desired computer-mediated communication use and workplace stress}

The stress literature emphasizes that demand conditions are subjectively appraised by individuals as stressors (Lazarus, 1990) in order for the individual to feel stressed. The same 'volume' of CMC use might influence the appraisal of workplace stress differently for different individuals (Karr-Wisniewski and Lu, 2010). Furthermore, individuals have various desires regarding how they wish to use CMC. These desires are often made salient when they are frustrated by organizational policies (Boell et al., 2016). For instance, despite problems of work-life conflict, work-life imbalance and burnout stemming from corporate smartphone use outside work (Derks et al., 2015), employees frequently desire to use these devices of their own free will in order to remain constantly available and display a greater sense of professionalism (Cavazotte et al., 2014). Similarly, positive views on email as a business critical tool have been found to lower feelings of email overload (Sumecki et al., 2011). We use the phrase 'desired CMC use' to describe how individuals would like to use CMC. 
Desired use often manifests in individuals' attitudes towards CMC. Individuals who feel apprehensive towards using CMC, avoid using them (Scott and Timmerman, 2005). Barley et al. (2011) showed that email use was not only a strong potential cause of workplace stress, but that it also "distracted people from recognizing other sources of overload in their work lives" (2011, p. 887). Participants focused so much on workplace stress due to email that the potential of other media to cause workplace stress was somehow overlooked. Viewing email negatively thus increased the appraisal of email being the cause of workplace stress (Barley et al., 2011).

However, there might be situations in which individuals do not have the option of using CMC as they desire. For instance, even though one might want fewer emails, reducing one's volume of email in the workplace might not be as easy or possible. The resulting misfits between one's actual and desired $\mathrm{CMC}$ use may influence the extent to which the individual appraises workplace stress. Such misfits have mostly been discussed in the form of mismatch in 'volume'. In particular, email overload has been defined as "users' perceptions that their own email use has" got out of control (Dabbish and Kraut, 2006, p. 431), which implies the importance of both the actual email volumes and the perceptions of these volumes.

The literature that we have discussed highlights that although CMC use can on its own influence workplace stress (E.g., Barley et al., 2011; Mano and Mesch, 2010), desired CMC use can affect this relationship. Those desiring greater (lesser) use of CMC could be less (more) stressed because of CMC use (Sumecki et al., 2011; Wright et al., 2014, Barley et al., 2011). Experience of workplace stress due to CMC use might thus be influenced by misfits between desired and actual use. 


\section{RESEARCH DESIGN}

We adopt a multi-methods approach in this study in order to examine these relationships between actual CMC use, desired CMC use and workplace stress. A multi-method design is defined as one that includes both quantitative and qualitative components (Creswell and Clark, 2011). The research problem we have identified is that of a lack of understanding of how actual and desired CMC use can together influence workplace stress. The multi-method design allows us to look at different facets (Mingers, 2001) of desired and actual CMC use affecting workplace stress together. We adopt a sequential multi-method design (Creswell and Clark, 2011), where a quantitative study precedes a qualitative one. Each study primarily answered one research question. The quantitative study allowed us to examine the extent to which actual and desired CMC use together influenced workplace stress, for a wide range of media and workplace stressor conditions. It thus mainly answered the first research question and also provided guidance for answering the second research question in the subsequent study. The qualitative study provided rich examples of how and why individuals' appraised workplace stressors was influenced by desired and actual CMC use together, mainly answering the second research question. It also partially contributed to the first research question by exploring in detail how the extent of use of different CMC media influenced different workplace stressors identified. The results of the first study thus feed the purpose and design of the second, consistent with sequential designs (Mingers, 2001). Both studies were analyzed separately.

In the next sections, we present the respective backgrounds and findings for each study separately, in their own parts. We also explain in more detail how and where each study's data were collected and analyzed. We then draw the contributions from each study into a merged, 
overall discussion as commonly done (E.g., Mann and Holdsworth, 2003) and methodologically recommended (Creswell and Clark, 2011).

\section{STUDY ONE - INVESTIGATING THE EXTENT TO WHICH ACTUAL AND DESIRED COMPUTER MEDIATED COMMUNICATION USE TOGETHER INFLUENCE WORKPLACE STRESS}

\subsection{Purpose}

Although workplace stress has been shown to be influenced by CMC use only (E.g., Barley et al., 2011; Mano and Mesch, 2010), we have highlighted literature showing that desired CMC use might also play an important role in this relationship. Literature has looked at the use of several different media such as email (E.g., Sumecki et al., 2011), instant messaging (Gupta et al., 2013) (Li et al., 2011) or social networks (E.g., Maier et al., 2015).

Given the diversity of both media and workplace stressors discussed in literature, there might be reasons to wonder if the extent of actual and desired CMC use together influence workplace stress, regardless of the CMC media and workplace stressors under study. Study 1 thus had the purpose of investigating the extent to which actual CMC use and desired CMC use together affected workplace stress, thereby primarily answering the first research question. In absence of specific guidance from the literature to the contrary, we hypothesize that workplace stress will be better explained by actual and desired CMC use together rather than by actual CMC use alone, for all media and workplace stressors.

Hypothesis: The variation in workplace stress will be explained significantly more by actual CMC use and desired CMC use together, than by actual CMC use alone. 


\subsection{Sample}

The sample for this study consisted of full-time U.S. workers recruited from a Qualtrics panel. This company was selected as the source of the sample due to the acknowledged quality of their samples and their prevalent use in academic research (Brandon et al., 2014). Qualtrics provided financial support for participant recruitment, but was not involved at any stage of the research. 795 individuals clicked on the link received by email and filled out the questionnaire. 67 of those were screened-out because they were not working full-time. Additionally, 2 participants were rejected on the basis that they answered four times quicker than the average answering time, and 222 because they failed to answer the attention filter question correctly. The attention filter question was "this is an attention filter, please answer "not at all"', and was placed in the middle of the questionnaire. Finally, 504 valid responses were collected with no missing data, which represents a usable response rate of $63 \%$. The sample was composed of $47.4 \%$ men and $52.6 \%$ women aged from 20 to 73 years, with a mean age of 44 years ${ }^{1}$.

\subsection{Measures}

\section{Independent variables: computer-mediated communication}

The first part of the questionnaire measured the extent of CMC use at work. This was assessed by the use of different media, included email, video conferencing, audio conferencing or phone calls and instant messaging. These media are commonly investigated together in studies of CMC (Scott and Timmerman, 2005). We also included enterprise social networking, which is a newer workplace medium inspired from social networking platforms. For each of these media, we asked participants to report the extents to which they (1) were interacting at work using each medium (i.e. actual CMC use), and (2) would like to interact at

\footnotetext{
${ }^{1}$ The full dataset is made freely available to readers who wish to refer to it.
} 
work using each medium (i.e. desired CMC use). All items were assessed using 7-point Likert scales ranging from " $1=$ Not at all" to " $7=$ To a very great extent".

\section{Dependent variables: workplace stress}

The second part of the questionnaire looked at workplace stress. This was assessed by the presence of several workplace stressors, using the 'A Shortened Stress Evaluation Tool' (Cartwright and Cooper, 2002; Faragher et al., 2004) that has been found reliable across multiple studies (Faragher et al., 2004; Johnson, 2009). These workplace stressors were work relationships (Cronbach $\alpha=.907,8$ items), work-life balance (Cronbach $\alpha=.790,4$ items), job security and change (Cronbach $\alpha=.811,4$ items), job conditions (Cronbach $\alpha=.771,9$ items), resources and communications (Cronbach $\alpha=.845,4$ items), job control (Cronbach $\alpha$ $=.896,4$ items), and workload (Cronbach $\alpha=.861,4$ items). Participants answered on a 5point Likert scale of agreement from 1="Strongly Disagree" to 5="Strongly Agree".

\subsection{Findings}

We hypothesized that workplace stress would be better explained by the influence of actual CMC use and desired CMC use together than by the influence of actual CMC use alone. The hypothesis was tested using hierarchical linear regressions for each workplace stressor as the dependent variable, for each medium. Hierarchical regressions were used in order to see the increment in variance explained by actual and desired CMC use together compared to actual CMC use alone. As shown in Table 1, the regression in Step 1 contained only the control variables. We selected age, gender, education, company size and persons under supervision as the control variables, as suggested in literature on workplace stress due to email (Mano and Mesch, 2010, p. 68). In Step 2, we added actual CMC use as an independent variable to the 
regression equation. Step 3 contained both actual and desired CMC use as independent variables.

The hypothesis is supported when the increase in $\mathrm{R}^{\mathbf{2}}$ (i.e. in predictive power) between Step 2 and Step 3 is significant, meaning that the model containing both actual and desired CMC use predicts stressors more than the model containing actual CMC use only. On the contrary, a significant increase in $\mathrm{R}^{2}$ between Step 1 and Step 2 but not between Step 2 and Step 3 would mean that workplace stress is better predicted by actual CMC use alone.

\section{$<$ INSERT TABLE 1 HERE >}

\section{Media and workplace stressors for which actual and desired use together, influenced workplace stress}

As shown in Table 1, the hypothesis was not supported for every communication medium and workplace stressor. The influence of actual and desired use together was found more strongly predictive of a number workplace stressors than the influence of actual use alone, for primarily email (See rows labeled "a" in Table 1). These included resources and communication stress, job control stress, relationship stress, workload stress, job security stress and job conditions stress.

This finding shows that the effect of actual and desired use together on workplace stress mostly concerns the use of the email medium. One possible explanation is that because it is the most commonly used CMC application, email is often considered as a "symbol of workplace stress" (Barley et al 2011); such a negative perception might act as a self-fulfilling prophecy and favor the appraisal of email being stressful (Sumecki et al., 2011). Another explanation could be that email is widely used and individuals are thus more likely to have 
precise desires regarding their email use regardless of their organization. The desired use for other media might not be very high, due the diversity of organizations present in the sample. Indeed, the mean extent of email use $(M=4.56)$ were much higher of those for the other $\operatorname{media}(M=[1.77,3.67])$.

\section{Media and workplace stressors for which actual use alone influenced workplace stress}

For some other media and workplace stressors, our hypothesis was not supported. An interesting relationship was however found. That is, actual CMC use alone, rather than actual and desired $\mathrm{CMC}$ use together, was significant in affecting workplace stress (See rows marked ' $b$ ' in Table 1).

Specifically work-life balance stress was positively influenced by CMC use alone for video conferencing, audio conferencing, instant messaging and enterprise social networking, as shown in the fourth row of each medium in Table 1. It might be that using these media increased the work-life balance stressor regardless of desired use, due to the difficulty of accessing them from outside work. Accessing the organization's social network, instant messaging system or video conferencing system is often a more stressful experience often requiring a virtual private network (VPN) or a laptop, and more time and isolation from the family environment, as compared to briefly checking emails on a corporate smartphone in "dead time" (Mazmanian et al., 2005, p. 3). Furthermore, when these media cannot be accessed from home, employees might have to stay longer at the office to use them, such as when a video conference or a phone call must be made with others across multiple time zones. This suggests that the use of non-email media might be related to work-life conflict regardless of desired use due to the complexity and uncertainty of operating these media outside work. 
The influence of CMC use alone was found significant for video conferences and relationship stress, workload, job security stress, as well as for enterprise social networks and relationship stress, workload stress, job security stress and job conditions stress. There could be two reasons for this. One, that use of video conferencing or enterprise social networks alone influenced these workplace stressors. Two, the participants may not have answered differently enough about their actual and desired use, perhaps because they did not have enough opinions about video conferencing and enterprise social networks due to their novelty and infrequent implementation in organizations. We note here that collinearity between actual and desired use for each medium $(\mathrm{VIF}=4.292)$ was at acceptable levels (Bowerman and O'Connell, 1990).

Finally, neither actual CMC use, nor actual and desired CMC use together, had any effect on the rest of the media - workplace stressors combinations. This is depicted in the rows in Table 1 unlabeled by either ' $a$ ' or ' $b$ '. This includes for example the effect of email use on work-life balance stress or the effect of instant messaging use on all workplace stressors but work-life balance.

The quantitative study primarily established that desired use and actual use influenced most workplace stressors together, but primarily for email. It thus suggested that the importance of desired CMC use depends on the particular media. For media other than email, we observed that actual CMC use alone had a significant effect on workplace stress. The absence of support for our hypothesis for media other than email might have been due to the variety of organizations investigated. In the second study we investigate the effect of actual and desired CMC use in one single organization. 


\section{STUDY TWO - EXPLORING HOW ACTUAL AND DESIRED CMC USE TOGETHER INFLUENCE WORKPLACE STRESS}

\subsection{Purpose}

Our quantitative study established that actual and desired CMC use can together influence workplace stress primarily for email, but was not able to explain how and why this was soThe purpose of Study 2 was therefore to explore how actual CMC use and desired CMC use can together influence workplace stress, in a single organization that had implemented all the media examined in Study 1. Specifically, we looked at situations in which actual and desired CMC use might interact or conflict, to influence workplace stress. We also explored whether desires were more salient for some media in particular, as was found in the preceding quantitative study. This study built on the quantitative study results in that participants were asked about the same media previously investigated and the initial list of codes was designed based on the quantitative findings.

\subsection{Study site, data collection and data analysis}

The setting for this study was a large multinational IT company. The organization employed a young workforce that used all the media we examined in our quantitative study, namely, email, instant messaging, phones, audio-video conferencing, wikis and enterprise social networking. 23 employees were interviewed at the organization's French headquarters. None of them participated in Study 1. Our interviewees, 17 men and 6 women, had a mean age of 32 years. 12 participants declared to have at least one person under their supervision, 5 had none but were experienced professionals, and 6 had none with entry-level jobs. We conducted the interviews based on an interview guide (Rubin and Rubin, 2011) of open-ended questions. Except for some preliminary demographical questions, the interview was semi-structured. The participants were asked about actual and desired CMC use, and about their influence on the 
workplace stressors ${ }^{2}$. These common questions helped to ensure comparability across the participants and their open-ended nature allowed emergence of unanticipated content. The interviews were conducted in French by the first author who is a French native speaker. Each interview lasted approximately 20 minutes and was recorded after the participant signed a consent form. The interviews were then transcribed and translated into English for analysis.

In order to analyze our interview data, an initial list of codes was drawn up ${ }^{3}$ based on the insights from Study 1, as well as from literature (E.g., Barley et al., 2011); the participants were given pseudonyms to safeguard their anonymity (see Table 2). The initial list of a priori codes included each medium and workplace stressor also present in the first study and the codes "Perceived fit" and "Perceived misfit". These a priori codes were "predetermined topic codes in the qualitative analysis that are based on the important factors identified in the quantitative results" (Creswell and Clark, 2011, p. 236); a practice consistent with our multimethod design. A few new codes emerged in the process, which were added to our initial list of codes. For instance, different types of fit between actual and desired CMC use were added. Some predetermined codes like job conditions stressor were dropped as they did not emerge during the interviews. Broadly described, the codes were grouped under the categories: actual CMC use, desired CMC use, workplace stress, and influence of actual and desired CMC use together on workplace stressors. The transcripts were read and coded according to this list.

\subsection{Findings}

We begin by noting that all media were not used to the same extent. Employees identified email as the most commonly used medium, followed by remote access and instant messaging.

\footnotetext{
${ }^{2}$ Interview schedule available on request.

${ }^{3}$ List of codes available on request.
} 
Participant responses revealed three key conditions through which actual and desired CMC use together influenced workplace stressors. The first condition was that of participants not having access to the CMC media they wanted to use for their work. The second was use of CMC media that they did not prefer, but imposed on them through organizational norms and policies. The third condition was colleagues imposing their desired CMC use, such that employees' own desired use conflicted with the desired use of others.

\section{Condition 1: Misfits between desired and available media}

We found that the actual and desired CMC use together increased workplace stress. However we obtained substantively richer and more granular insights, over and above the statistical relationships in Study 1. For instance, employees who felt they had access to the 'right' kind of media or combination of media, felt less exposed to workplace stress. Participants articulated that the range of media they had in their jobs made them whole and allowed them to interact smoothly and without stress, because it gave them access to a wide array of means of communication that they wanted to use. This was made salient by Gabe, a manager in his early thirties who tried to use all media at his disposal. When asked about additional media that he could have used, Gabe declared: 'I don't feel there is a missing link'. He was satisfied with the range of media he had access to because in that range he could find the ones he wanted to use. Judith, who recently switched to part-time work, explained that the corporate smartphone she asked to use allowed her to switch from home matters to work matters in a smooth and soothing way:

"My children were playing football. I took my Blackberry and answered emails for a few minutes. It felt good. [...] I told them 'I won't be with you for ten minutes, I will be in my own bubble' and that was it". 
She declared earlier: 'these media make my life easier. Honestly, they are not a source of stress but of tranquility.' By being allowed to access emails from home, Judith felt a sense of flow or completeness that could be termed as 'media fit'. Mazmanian and colleagues (2005) have already highlighted such productive use of "dead times", but the satisfaction Judith expressed was also the result of being allowed to access to the desired medium at the appropriate time.

A sense of misfit appeared when the need for a specific medium was not met. This had similarities with the concept of task-technology fit (Goodhue and Thompson, 1995), which is in part, about having access to the technologies that fit the employees' workflows and are needed. Some participants complained about lack of specific media. Roy, whose role is to provide real-time technical support to other employees, was annoyed that users were not using the ticketing system enough and used emails or instant messages to contact him instead. Although minor, these annoyances seemed to contribute to a sense of increased workload. This type of frustration might also be related to studies showing that new media such as instant messaging, social networks or ticketing systems compete with older media such as emails to fulfill individuals' needs and demands (c.f. Ramirez et al., 2008).

\section{Condition 2: Misfits between desired and imposed CMC use}

Our study participants frequently discussed how the use of particular media was imposed on them by the organization or by colleagues. This mostly took the form of interruptions and notifications of incoming messages to which employees were expected to respond even if they did not want to. Russell, a manager who used to work abroad for a time, compared these interruptions with the action of 'tapping you on the shoulder virtually', as he found them as disturbing as physical interruptions. Roy, the employee in the frontline technical support function, declared having tried a small experiment to calculate the frequency at which he was 
being interrupted by other employees. He tried to listen to a three-minute song but was never able to finish listening to it without being interrupted by an instant message or an email from someone asking for his help. The consequences of such interruptions could include higher perceived workload and workplace stress (c.f. Barber and Santuzzi, 2015; Gupta et al., 2013).

To fight these constant interruptions, techniques were suggested. Roy and others sometimes logged out of the instant messaging system, deactivated the email notification pop-ups, or tried to put a 'busy' status on the instant messaging. Peter, who declared being a 'people's person', sometimes nevertheless 'played dead' and faked being away by not responding. This generally failed as the frustrated senders decided to come to see Peter, Roy and others face-toface instead, thus interrupting them anyway.

Even the technique of slowing the pace of email delivery did not prevent such behaviors. Carl had been successful at doing this in several other companies where he had worked previously, but was not able to make it work in the current one:

"[In my previous job] I had my software retrieve emails every hour or every hour and a half, so I was not interrupted for an hour. Here in this company, I don't do this because even when there are no emails, there is [the instant messaging system] and people coming to see you at your desk, so..."

Some respondents also explained how they used various media to impose communications on others. Gabe, the manager who earlier declared being satisfied with the media at his disposal, was nevertheless a heavy user of email. One reason why he liked email in particular was that 'emails are pushed... Since I impose them on others, I know that they will be read'. Although the more senior managers might have more power to impose their desired communications onto others (Gupta et al., 2013), others successfully imposed their communications as well. 
Natalie, despite being a young entry-level accountant with no managerial responsibilities, discussed her technique to get prompt answers: 'After two hours, I log into the instant messaging system and I send a message 'so have you seen my email?' I think it puts a bit of pressure [laughs]'. Natalie was clearly able to impose her communications onto others despite her young age and lack of managerial responsibilities. These cases interestingly highlight that interruptions disturbing some employees are created by others in the first place.

\section{Condition 3: Misfits between one's own desired CMC use and those of others}

An imposed interaction through CMC use by someone else was found to be disturbing and annoying. This was especially the case when employees had to work together had different desired CMC use. In such situations, the desired CMC use of one employee could very well conflict with that of another. Michael, a manager in his late thirties, had to use a large variety of media to communicate with his team on-site and abroad. Yet in this large variety of media, he strongly disliked one in particular:

“I limit my phone calls a lot. I never call. I don't like the phone, I don’t know why [laughs]. I don't like it, and I don't like it either when I am called.”

On the other hand, Peter, the people person who sometimes 'played dead' to avoid instant messages, said:

"I am more of a phone person. [...] I send an instant message with 'hey, do you have a minute?" and if they say yes, I call them straight away. I think people have identified me as someone who calls [laughs]."

Perhaps because Peter did not enjoy being interrupted by instant messages, he always warned his colleagues that he was about to call them. He used instant messages as a buffer to transition into the medium he really enjoyed using: the phone. Although Peter and Michael were not in the same team and hence did not have to put up with the desires of one another, 
they each had to deal with their own desires. Michael never expressed his dislike of the phone to anyone. He just lived with it. Peter claimed that others knew his phone preference, but were not bothered by it, especially as he refrained from calling them if hisinstant message sent beforehand was not answered.

There was therefore a misfit between Peter's and Michael's desired use of phone calls and the actual use that was possible in their jobs, due to different desired use of colleagues. Research has examined the concept of 'supplementary' fit. Supplementary fit occurs when an individual does not possess preferences or desires which are similar to those of others in the same environment (Muchinsky and Monahan, 1987, p. 269). Thus, employees having similar desires in terms of CMC or media use, or greater supplementary fit, might have less stressful interactions with one another. For instance, employees who enjoy constant connectivity to work through the smartphone might be able to work well with supervisors who share such preferences (Derks et al., 2015).

Therefore, being able to understand the desired CMC use of colleagues is a useful skill for employees to have. As one participant described, 'it is about adapting the medium to the person'. Some individuals were particularly aware that others knew their desired CMC use. Just as Peter who preferred phone communication in our example above, was identified 'as someone who calls', Russel, a former virtual worker, said 'I've been here almost three years now. I think people are aware of the best way to get hold of me'. That said however, being able to understand the desired CMC use of work colleagues is not always easy. It requires understanding of various media as well as empathy, both of which might be hard to come by: "It is hard for me to tell because I have been using these technologies for a very long time. They have become so natural that it is sometimes hard for me to realize that they might not be 
as natural to others." (Jerry, a manager in his late twenties). In studies of work-life conflict and smartphone use, managers are often urged to explicitly share their expectations regarding CMC use, such as constant availability. Not doing so, could inadvertently damage their subordinates' work-life balance (Derks et al., 2015). Through these findings we thus identify three conditions illustrating the joint influence of actual and desired CMC use on workplace stress. We next discuss the implications of our two studies.

\section{DISCUSSION AND CONCLUSION}

\subsection{Multi-method results}

In this section, we discuss how the combined findings of the two studies helped generate a richer understanding of how and why employees experience workplace stress due to the use of CMC at work, beyond that of each study alone (See Figure 1).

\section{< INSERT FIGURE 1 HERE >}

First, with regard to which medium was found to most influence workplace stress, both studies revealed the prominence of email use. The qualitative study showed that although the interviewees worked in a company which was fairly advanced in terms of CMC implementation, they mainly discussed their use of email, and the workplace stress they attributed to its use. In the qualitative study, however, participants also discussed how their workplace stress was influenced by their desired use of other media widely used in the company such as instant messaging and audio-video conferences. From the quantitative study we infer that both desired and actual use can cause for workplace stress, for those media that were highly used. For other media that are not widely used, the extent of use alone is a cause of workplace stress. In addressing our first research question therefore, we note that desired CMC use is more salient and relevant to workplace stress mostly for those media which are 
widely used. The organizational context in which social norms are developed over time regarding to what extent specific media are used might thus play an important role in the influence of desired CMC use on workplace stress.

Regarding the second research question, the qualitative study found three conditions under which actual and desired CMC use together influenced workplace stress. First, participants expressed frustration when they were forced to use media inappropriate to the task at hand or when alternative media considered more appropriate were not available. Second, imposed interactions and unwanted interruptions regarding use of media that employees were reluctant to use, were considered stressful. Finally, workplace stress from CMC use was experienced by employees who interacted with co-workers possessing different or conflicting preferences for using media than they did.

The limitations of the studies must be kept in mind when reflecting on our results. The quantitative study included participants from diverse organizations and industries, and measured a number of variables, some relying on single items. Although this revealed interesting results such as the importance of actual and desired use of email, due to its wide sampling, it might have also downplayed or reduced the significance of other media. Furthermore, the qualitative study used a sample unrelated to the quantitative one, which, while providing the opportunity to triangulate and identify integrative findings, makes direct comparison between the two sets of results difficult. Notwithstanding these limitations, the paper makes important contributions to literature and practice, which we discuss below. 


\subsection{Theoretical contributions and future research}

The paper's first contribution is in revealing that all media are not the same with regard to the phenomenon of stress that employees experience from their use in the workplace. We found that the influence of actual and desired CMC use, together, on workplace stress was significant mostly for email, in the quantitative study. In the qualitative study, participants reinforced the importance of email by articulating how their desired use was important to understand the process of workplace stress associated with their use of email. Literature shows that email is associated with strong attitudes regarding workplace stress from its use (Barley et al., 2011). Our study extends this notion to suggest that workplace stress from email use is more influenced by the individual's preference for using email, in comparison with other media. We thus suggest that media that are more widely used, should be designed as to take into account the individual's preferences and choices regarding use (E.g., Stacey and Tether, 2015, p. 114). One avenue for future research that naturally suggests itself from this finding is the investigation of separate media through separate nomological models in terms of their users, workplace stress generating potential, and circumstance of use. This would be in contrast to the existing models of CMC research which aggregate use measures of various media.

Our second theoretical contribution is in articulating the concept of 'misfit' in the context of workplace stress from the use of CMC. The fit between the individual and the environment has been investigated under the purview of person-environment fit theories (Kristof-Brown et al., 2005). Person-environment fit has been defined as "the compatibility between an individual and a work environment that occurs when their characteristics are well matched" (Kristof-Brown et al., 2005, p. 281). Such compatibility increases positive work outcomes such as job satisfaction, job engagement and satisfaction with coworkers. 'Misfit' or a lack of 
compatibility reduces these outcomes. Drawing on these concepts, we make a theoretical contribution by showing that lack of fit can manifest in the form of three conditions, which embody misfit. The presence of these conditions is an indication of misfit between the way in which the individual desires to use media, and actually uses them, and creates workplace stress for the individual. We believe this to be an interesting and new conceptual area for future research on CMC use and workplace stress.

Our third contribution is in focusing attention on the individual's desires with regard to CMC use. While the literature informs us that use of media, particularly email, can cause workplace stress for the user, it does not explain how this relationship may be individual specific. We show different facets of the individual's desires regarding CMC use that are important in this context. The quantitative study revealed that the extent of actual use and desired use together influence workplace stress. The qualitative study showed that in addition to desired CMC use, other aspects such as the type of media desired to be used, other peoples' desires regarding media to be used, and the organization's norms regarding which CMC should be used and how, determine the workplace stress from CMC use. These findings open a new conceptual direction in CMC research, which has so far focused mainly on the extent of use.

\subsection{Practical implications}

Our findings also have practical contributions both for individuals and organizations. Individuals and especially those frequently initiating communications or having managerial responsibilities (Gupta et al., 2013) have a special responsibility in terms of others' workplace stress. By imposing their messages and favorite media onto others who might have different desired CMC use, they risk creating 'misfit', hereby worsening workplace stress due to CMC 
use. The fit of one could cause the misfit of another. This warning emphasizes the importance of having empathy for others' desired CMC use as well as knowing one's own.

Organizations might also benefit from our findings by investigating which media are widely used by their employees and by considering their employees' desired CMC use. Results from such studies could be collectively discussed with employees (Barber and Santuzzi, 2015), thereby making employees more aware of their own desired CMC use. As such, refusing to implement media widely desired by employees has the potential of creating misfit, hereby increasing workplace stress. On the contrary, imposing media that are widely rejected by employees has the similar potential to increase workplace stress. Finally, our multi-methods approach emphasized that there might not be a 'one size fits all' solution and that each organization and individual should try to apply our findings to their own contexts. 


\section{REFERENCES}

Ayyagari, R. (2012), 'Impact of Information Overload and Task-technology Fit on Technostress', SAIS 2012 Proceedings.

Barber, L. K. and Santuzzi, A. M. (2015), 'Please Respond ASAP: Workplace Telepressure and Employee Recovery', Journal of Occupational Health Psychology, 20, 2, 172189.

Barley, S. R., Meyerson, D. E. and Grodal, S. (2011), 'E-mail as a Source and Symbol of Stress', Organization Science, 22, 4, 887-906.

Boell, S. K., Cecez-Kecmanovic, D. and Campbell, J. (2016), 'Telework paradoxes and practices: the importance of the nature of work', New Technology, Work and Employment, 31, 2, 114-131.

Bowerman, B. L. and O'Connell, R. T. (1990), Linear statistical models: An applied approach. (PWS-Kent Boston).

Brandon, D. M., Long, J. H., Loraas, T. M., Mueller-Phillips, J. and Vansant, B. (2014), 'Online Instrument Delivery and Participant Recruitment Services: Emerging Opportunities for Behavioral Accounting Research', Behavioral Research in Accounting, 26, 1, 1-23.

Byron, K. (2008), 'Carrying Too Heavy a Load? The Communication and Miscommunication of Emotion by Email', Academy of Management Review, 33, 2, 309-327.

Cartwright, S. and Cooper, C. L. (2002), 'ASSET: Management guide'. (RobertsonCooper Ltd, UK).

Cavazotte, F., Heloisa Lemos, A. and Villadsen, K. (2014), 'Corporate smart phones: professionals' conscious engagement in escalating work connectivity', New Technology, Work and Employment, 29, 1, 72-87.

Cooper, C. L., Dewe, P. J. and O’Driscoll, M. P. (2001), Organizational stress: A review and critique of theory, research, and applications. (SAGE Publications).

Creswell, J. W. and Clark, V. L. P. (2011), Designing and Conducting Mixed Methods Research. (SAGE). 
Dabbish, L. A. and Kraut, R. E. (2006) 'Email overload at work: an analysis of factors associated with email strain', in: Proceedings of the 2006 20th anniversary conference on Computer supported cooperative work. pp. 431-440.

Dén-Nagy, I. (2014), 'A double-edged sword?: a critical evaluation of the mobile phone in creating work-life balance', New Technology, Work and Employment, 29, 2, 193-211.

Derks, D., Bakker, A. B., Peters, P. and Wingerden, P. van (2016), 'Work-related smartphone use, work-family conflict and family role performance: The role of segmentation preference', Human Relations, 69, 5, 1045-1068.

Derks, D., van Duin, D., Tims, M. and Bakker, A. B. (2015), 'Smartphone use and workhome interference: The moderating role of social norms and employee work engagement', Journal of Occupational and Organizational Psychology, 88, 1, 155177.

Faragher, E. B., Cooper, C. L. and Cartwright, S. (2004), 'A shortened stress evaluation tool (ASSET)', Stress and Health, 20, 4, 189-201.

Goodhue, D. L. and Thompson, R. L. (1995), 'Task-technology fit and individual performance', MIS Quarterly, 213-236.

Gupta, A., Li, H. and Sharda, R. (2013), 'Should I send this message? Understanding the impact of interruptions, social hierarchy and perceived task complexity on user performance and perceived workload', Decision Support Systems, 55, 1, 135-145.

Higgins, C. A., McClean, R. J. and Conrath, D. W. (1985), 'The accuracy and biases of diary communication data', Social Networks, 7, 2, 173-187.

Johnson, S. (2009) 'Organizational Screening: The ASSET Model', in: Cartwright, S. and Cooper, C. L. (eds.) The Oxford handbook of organizational well-being. (Oxford: Oxford University Press),

Karr-Wisniewski, P. and Lu, Y. (2010), 'When more is too much: Operationalizing technology overload and exploring its impact on knowledge worker productivity', Computers in Human Behavior, 26, 5, 1061-1072.

Kristof-Brown, A. L., Zimmerman, R. D. and Johnson, E. C. (2005), 'Consequences of Individuals' Fit at Work: A Meta-Analysis of Person-Job, Person-Organization, Person-Group, and Person-Supervisor Fit', Personnel Psychology, 58, 2, 281-342. 
Lazarus, R. S. (1990), 'Theory-Based Stress Measurement', Psychological Inquiry, 1, 1, 313.

Li, H., Gupta, A., Luo, X. and Warkentin, M. (2011), 'Exploring the impact of instant messaging on subjective task complexity and user satisfaction', European Journal of Information Systems, 20, 2, 139-155.

Maier, C., Laumer, S., Eckhardt, A. and Weitzel, T. (2015), 'Giving too much social support: social overload on social networking sites', European Journal of Information Systems, 24, 5, 447-464.

Mann, S. and Holdsworth, L. (2003), 'The psychological impact of teleworking: stress, emotions and health', New Technology, Work \& Employment, 18, 3, 196.

Mano, R. S. and Mesch, G. S. (2010), 'E-mail characteristics, work performance and distress', Computers in Human Behavior, 26, 1, 61-69.

Mazmanian, M., Orlikowski, W. J. and Yates, J. (2005) 'Crackberries: The social implications of ubiquitous wireless e-mail devices', in: Designing ubiquitous information environments: Socio-technical issues and challenges. (Springer), pp. 337-343.

Mingers, J. (2001), 'Combining IS research methods: towards a pluralist methodology', Information Systems Research, 12, 3, 240-259.

Muchinsky, P. M. and Monahan, C. J. (1987), 'What is person-environment congruence? Supplementary versus complementary models of fit', Journal of Vocational Behavior, 31, 3, 268-277.

Ramirez, A., Dimmick, J., Feaster, J. and Lin, S.-F. (2008), 'Revisiting Interpersonal Media Competition The Gratification Niches of Instant Messaging, E-Mail, and the Telephone', Communication Research, 35, 4, 529-547.

Rubin, H. J. and Rubin, I. S. (2011), Qualitative interviewing: The art of hearing data. (Sage Publications).

Scott, C. R. and Timmerman, C. E. (2005), 'Relating Computer, Communication, and Computer-Mediated Communication Apprehensions to New Communication Technology Use in the Workplace', Communication Research, 32, 6, 683-725.

Stacey, P. K. and Tether, B. S. (2015), 'Designing emotion-centred Product Service Systems: The case of a cancer care facility', Design Studies, 40, 85-118. 
Stich, J.-F., Farley, S., Cooper, C. and Tarafdar, M. (2015), 'Information and communication technology demands: outcomes and interventions', Journal of Organizational Effectiveness: People and Performance, 2, 4, 327-345.

Sumecki, D., Chipulu, M. and Ojiako, U. (2011), 'Email overload: Exploring the moderating role of the perception of email as a 'business critical' tool', International Journal of Information Management, 31, 5, 407-414.

Wright, K. B., Abendschein, B., Wombacher, K., O’Connor, M., Hoffman, M., Dempsey, M., et al. (2014), 'Work-Related Communication Technology Use Outside of Regular Work Hours and Work Life Conflict The Influence of Communication Technologies on Perceived Work Life Conflict, Burnout, Job Satisfaction, and Turnover Intentions', Management Communication Quarterly, 28, 4, 507-530. 


\section{TABLES}

Table 1: Results of hierarchical regressions and changes in $R$-squared values

\begin{tabular}{|c|c|c|c|c|c|c|}
\hline Medium & Workplace Stressor & $\begin{array}{c}\text { Step1. } \\
\mathrm{R}^{2} \\
\text { Control }\end{array}$ & $\begin{array}{c}\text { Step } 2 . \\
\mathrm{R}^{2} \\
\text { Actual }\end{array}$ & $\begin{array}{l}\text { Step 3. } \mathrm{R}^{2} \text { Actual, } \\
\text { Desired }\end{array}$ & $\begin{array}{c}\Delta \mathrm{R}^{2} \\
\text { Step1- } \\
\text { Step2 }\end{array}$ & $\begin{array}{c}\Delta \mathrm{R}^{2} \\
\text { Step2- } \\
\text { Step 3 }\end{array}$ \\
\hline \multirow{7}{*}{ E-mail } & Resources and Communication ${ }^{a}$ & .011 & .013 & $.045 * * *$ & .002 & $.032 * * *$ \\
\hline & Control $^{\mathrm{a}}$ & .013 & .020 & $.066^{* * *}$ & .007 & $.046^{* * * *}$ \\
\hline & Work Relationships ${ }^{\mathrm{a}}$ & .023 & .027 & $.060^{* * *}$ & .004 & $.033 * * *$ \\
\hline & Work Life Balance & $.045^{* * *}$ & .045 & .052 & .000 & .007 \\
\hline & Workload $^{\mathrm{a}}$ & .005 & .006 & $.026^{* * * *}$ & .001 & $.035 * * *$ \\
\hline & Job Security \& Change ${ }^{a}$ & .021 & .027 & $.060^{* * *}$ & .006 & $.033 * * *$ \\
\hline & Job Conditions $^{\mathrm{a}}$ & $.031 * *$ & $.047 * *$ & $.066^{* * *}$ & $.016^{* *}$ & $.019 * *$ \\
\hline \multirow{7}{*}{$\begin{array}{l}\text { Video } \\
\text { conference }\end{array}$} & Resources and Communication & .011 & .013 & .013 & .002 & .000 \\
\hline & Control & .013 & .013 & .013 & .000 & .000 \\
\hline & Work Relationships ${ }^{\mathrm{b}}$ & $.023^{*}$ & $.035^{*}$ & $.036^{*}$ & $.012 *$ & .001 \\
\hline & Work Life Balance ${ }^{\mathrm{b}}$ & $.045^{* * *}$ & $.095 * * *$ & $.097 * * *$ & $.050 * * *$ & .002 \\
\hline & Workload $^{\mathrm{b}}$ & .005 & $.040 * * *$ & $.040^{* * *}$ & $.035 * * *$ & .000 \\
\hline & Job Security \& Change ${ }^{b}$ & .021 & $.033^{*}$ & $.035^{*}$ & $.012 *$ & .002 \\
\hline & Job Conditions & $.031 * *$ & .035 & .035 & .004 & .000 \\
\hline \multirow{7}{*}{$\begin{array}{c}\text { Audio } \\
\text { conference }\end{array}$} & Resources and Communication ${ }^{\mathrm{a}}$ & .011 & .011 & .020 & .000 & $.009 *$ \\
\hline & Control $^{\mathrm{a}}$ & .013 & .017 & $.030 *$ & .004 & $.013 *$ \\
\hline & Work Relationships ${ }^{\mathrm{a}}$ & $.023 *$ & .024 & .027 & .001 & .003 \\
\hline & Work Life Balance $^{\mathrm{b}}$ & $.045^{* * *}$ & $.057 *$ & $.062 *$ & $.012 *$ & .005 \\
\hline & Workload & .005 & .009 & .016 & .004 & .007 \\
\hline & Job Security \& Change & .021 & .021 & .022 & .000 & .001 \\
\hline & Job Conditions & $.031 * *$ & .031 & .037 & .000 & .006 \\
\hline \multirow{7}{*}{$\begin{array}{c}\text { Instant } \\
\text { Messaging }\end{array}$} & Resources and Communication & .011 & .011 & .011 & .000 & .000 \\
\hline & Control & .013 & .016 & .016 & .003 & .000 \\
\hline & Work Relationships & $.023^{*}$ & .023 & .024 & .000 & .001 \\
\hline & Work Life Balance $^{\mathrm{b}}$ & $.045^{* * *}$ & $.069^{* * *}$ & $.069 * *$ & $.024 * * *$ & .000 \\
\hline & Workload & .005 & .005 & .012 & .000 & .007 \\
\hline & Job Security \& Change & .021 & .023 & .023 & .002 & .000 \\
\hline & Job Conditions & $.031 * *$ & .031 & .032 & .000 & .001 \\
\hline \multirow{7}{*}{$\begin{array}{c}\text { Enterprise } \\
\text { Social } \\
\text { Networking }\end{array}$} & Resources and Communication & .011 & .014 & .017 & .003 & .003 \\
\hline & Control & .013 & .013 & .015 & .000 & .002 \\
\hline & Work Relationships ${ }^{\mathrm{b}}$ & $.023^{*}$ & $.039 * *$ & $.041 * *$ & $.016 * *$ & .002 \\
\hline & Work Life Balance $^{\mathrm{b}}$ & $.045^{* * *}$ & $.095 * * *$ & $.097 * * *$ & $.050 * * *$ & .002 \\
\hline & Workload $^{\mathrm{b}}$ & .005 & $.042 * * *$ & $.042 * * *$ & $.037 * * *$ & .000 \\
\hline & Job Security \& Change ${ }^{b}$ & .021 & $.031^{*}$ & $.037 *$ & $.010 *$ & .006 \\
\hline & Job Conditions ${ }^{\mathrm{b}}$ & $.031 * *$ & $.038^{*}$ & .042 & $.007 *$ & .004 \\
\hline
\end{tabular}

$* p<.05 ; * * p<.01 ; * * * p<.001$

${ }^{a}$ The hypothesis is supported: actual and desire use both influence the workplace stressor

${ }^{\mathrm{b}}$ The hypothesis is not supported but post-hoc analyses reveal that actual use alone influence the workplace stressor 
$\Delta R^{2} X-Y$ : Change in $R$-squared between Step X and Step $Y$

Table 2: Table of Interviewees

\begin{tabular}{cc}
\hline Pseudonym & Demographics \\
\hline Gabe & Male, 32, Front Line Manager \\
Judith & Female, 35, Experienced non manager, part-timer \\
Roy & Male, 32, Front Line Manager \\
Russell & Male, 31, Front Line Manager \\
Peter & Male, 30, Front Line Manager \\
Carl & Male, 30, Experienced non manager \\
Philip & Male, 27, Entry level \\
Natalie & Female, 31, Entry level \\
Michael & Male, 36, Front Line Manager \\
Jerry & Male, 27, Front Line Manager \\
\hline
\end{tabular}




\section{Figures}

Figure 1: Findings of both studies on the influence of actual and desired CMC use on workplace

stress

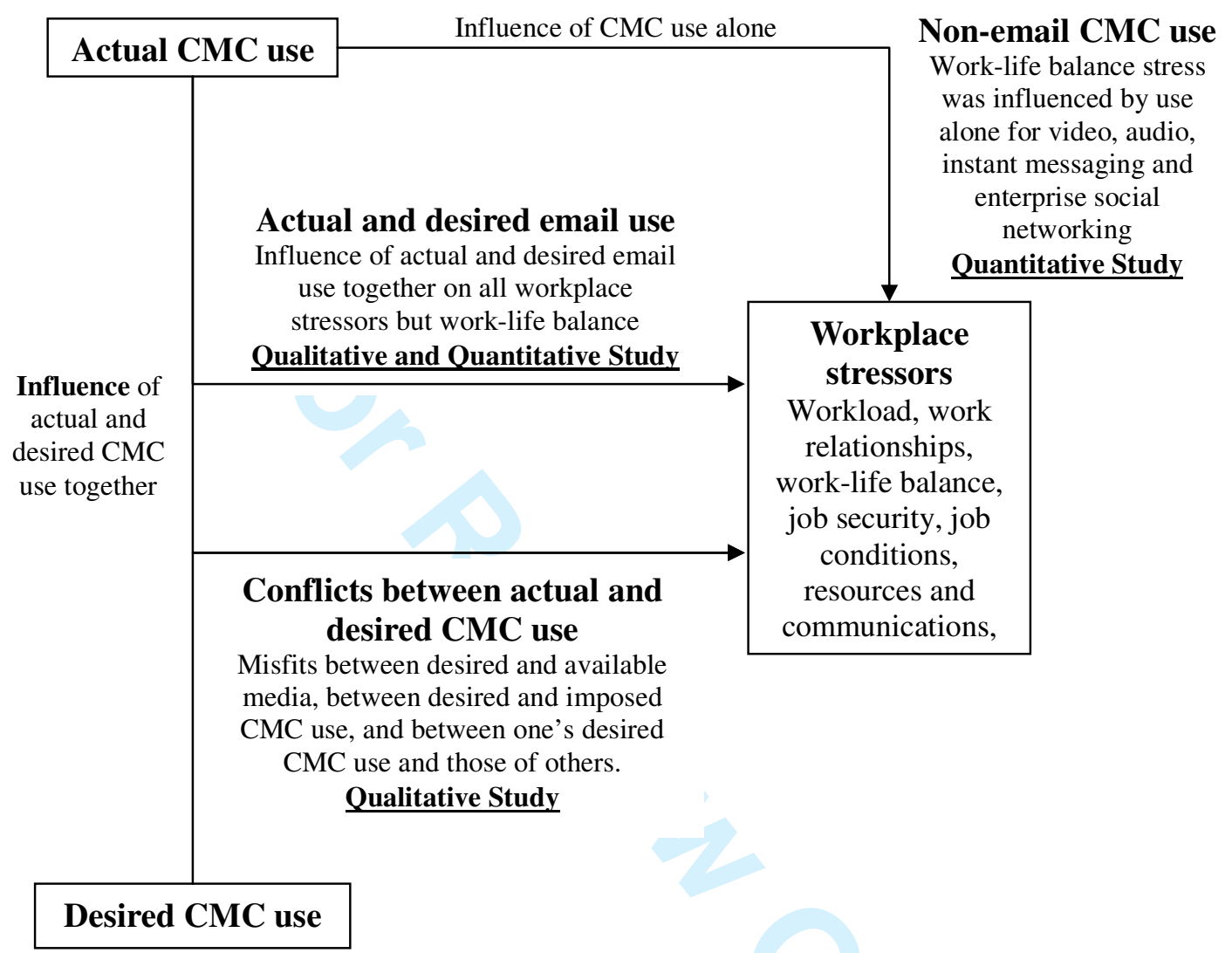

